\title{
On the Thermal Gauge Boson Masses of the Electroweak Theory in the Broken Phase
}

\author{
Cristina Manuel \\ Dpt. Estructura i Constituents de la Matèria \\ Facultat de Física, Universitat de Barcelona \\ Diagonal 647, 08028 Barcelona (SPAIN)
}

\begin{abstract}
Thermal effects in the broken phase of the electroweak theory are studied in the strongly interacting Higgs boson limit. In that limit and at tree level the bosonic sector of the theory is a gauged non-linear sigma model. The associated one-loop thermal effective action for soft fields is then computed by using the background field method together with the Stueckelberg formalism. This effective action describes thermal corrections to the masses of the gauge bosons $W, Z$ and the photon. It is the proper generalization of the hard thermal effective action of a Yang-Mills theory when there is a Higgs mechanism for a heavy Higgs particle.
\end{abstract}

PACS No: 11.10.Wx, 12.15-y, 12.38.Bx, 12.20.Ds

ECM-UB-PF-98/01

January/1998 


\section{INTRODUCTION}

This article is devoted to study thermal effects in the broken phase of the electroweak model in the strongly interacting Higgs boson limit. In that limit the Higgs field becomes heavy and it can be integrated out. At tree level the corresponding low energy effective theory is a gauged non-linear sigma model [1].

The electroweak theory is supposed to undergo a phase transition at high temperature $T$ [2]. The fact that the model has a completely different behavior at low or high temperatures has important phenomenological consequences, which will not be discussed here. Instead, I will compute one-loop thermal effects at $T$ far below the electroweak phase transition, taking profit of some results already obtained in the contexts of QCD and ungauged nonlinear sigma models. Let us first comment about those two different field theories.

In the last few years much progress has been made in understanding the leading thermal effects in non-Abelian gauge theories, such as QCD [3]. Even if at $T=0$ the non-Abelian gauge fields are massless, at finite $T$ there is a thermal mass, the so-called Debye mass, for non-Abelian electric fields. The Debye mass can be computed at one-loop order in perturbation theory. To take into account the thermal effects of Debye screening properly a resummation of an infinite number of Feynman diagrams, the hard thermal loops (HTL's), is required [4], [5], [6]. Hard thermal loops are thermal amplitudes which arise in a gauge theory when the external loop momenta is soft, while the internal one is hard. Soft denotes a scale $\sim g T$, while hard refers to one $\sim T$, where $g \ll 1$ is the gauge coupling constant. The effective action generating HTL's is a mass term for the chromoelectric fields, and it has been constructed just by solving a gauge invariance condition imposed on it [6]. This mass term is, however, non-local.

When a global $S U(N)_{L} \times S U(N)_{R}$ symmetry is spontaneously broken to $S U(N)_{L+R}$ the physics of the corresponding Goldstone bosons at lowest order in their momenta is described by the (ungauged) non-linear sigma model. Thermal effects in this model have already been considered in the literature [7], [8], [9], [10]. Surprisingly, the same one-loop thermal amplitudes as before, the HTL's, appear in the framework of the non-linear sigma model [9], [10]. This can be understood in terms of symmetry arguments. In this case HTL's give account of thermal scattering among the Goldstone bosons.

I will use the knowledge of the thermal effects of the two above mentioned models for the low energy effective theory of the electroweak model. The one-loop thermal effective action for soft gauge fields in this effective model will be computed. For the gauge fields to be soft, one has to require that the masses of the gauge bosons be also soft, thus $\ll T$. The temperature range of validity of the results is then $M_{W}, M_{Z} \ll T \ll \sqrt{12} v$, where $M_{W}$ and $M_{Z}$ are the masses of the gauge bosons $W$ and $Z$, respectively, and $v$ is the vacuum expectation value of the Higgs doublet at $T=0$. The upper limit is so because in the $N=2$ non-linear sigma model thermal corrections can be written as an expansion in the dimensionless parameter $\left(T^{2} / 12 v^{2}\right)$.

The computation will be done using the background field method [1] together with the Stueckelberg formalism [12], [13]. The thermal effective action computed here turns out to be, in the unitary gauge, a combination of the one-loop thermal effective actions which appear, on one hand, in a pure Yang-Mills theory, and on the other hand, in a non-linear 
sigma model in the presence of external currents. The result is thus written in terms of the same non-local thermal effective actions which appear in the previous mentioned theories. Furthermore, it gives a description of the thermal corrections to the masses of the gauge bosons. Therefore, it can be considered as the proper generalization of the hard thermal loop effective action of a non-Abelian gauge field theory when there is a Higgs mechanism, and for a heavy Higgs particle.

This paper is structured as follows. In Sect. 四 the bosonic sector of the electroweak model is reviewed. The corresponding effective theory in the strongly interacting Higgs boson limit is a gauged non-linear sigma model. In Sect. [II the background field method and the Stueckelberg formalism applied to that model are presented. In Sect. IV the oneloop thermal effective action is obtained. From that action the thermal corrections to the masses of the gauge bosons are extracted in Sect. D. The last Section is devoted to the conclusions.

\section{BOSONIC SECTOR OF THE ELECTROWEAK THEORY}

In order to set up the notation and conventions used in this article the bosonic sector of the electroweak theory is briefly reviewed in this Section [1]. The classical Lagrangian of the bosonic sector of the electroweak theory $S U(2)_{W} \times U(1)_{Y}$ is written in terms of the weak isospin gauge field $W_{\mu}$, the weak hypercharge gauge field $B_{\mu}$, and the complex scalar doublet $\Phi$. Using the standard linear representation for the Higgs fields, the classical Lagrangian reads

$$
\begin{aligned}
\mathcal{L}_{c l} & =\mathcal{L}_{\text {gauge }}+\mathcal{L}_{\text {Higgs }} \\
& =-\frac{1}{2} \operatorname{Tr}\left(W_{\mu \nu} W^{\mu \nu}\right)-\frac{1}{4} B_{\mu \nu} B^{\mu \nu}+D_{\mu} \Phi D^{\mu} \Phi^{\dagger}+\mu^{2} \Phi \Phi^{\dagger}-\lambda\left(\Phi \Phi^{\dagger}\right)^{2},
\end{aligned}
$$

where the $S U(2)_{W}$ and $U(1)_{Y}$ field strength are

$$
W_{\mu \nu}=\partial_{\mu} W_{\nu}-\partial_{\nu} W_{\mu}+i g\left[W_{\mu}, W_{\mu}\right], \quad B_{\mu \nu}=\partial_{\mu} B_{\nu}-\partial_{\nu} B_{\mu},
$$

with $W_{\mu}=\frac{1}{2} \vec{W}_{\mu} \cdot \vec{\tau}$, and $\vec{\tau}=\left(\tau^{1}, \tau^{2}, \tau^{3}\right)$ are the Pauli matrices. The covariant derivative acting on the Higgs doublet is

$$
D_{\mu} \Phi=\partial_{\mu} \Phi+i g W_{\mu} \Phi+i \frac{g^{\prime}}{2} B_{\mu} \Phi .
$$

The complex $S U(2)_{W}$ doublet is represented linearly as

$$
\Phi(x)=\left(\begin{array}{c}
\varphi^{+}(x) \\
\varphi^{0}(x)
\end{array}\right) .
$$

To study the strongly interacting effects of the electroweak model it is more convenient to use a different representation for the Higgs sector. After defining the matrix [i]]

$$
M(x)=\sqrt{2}\left(\begin{array}{cc}
\varphi^{0 \dagger}(x) & \varphi^{+}(x) \\
-\varphi^{-}(x) & \varphi^{0}(x)
\end{array}\right)=\sqrt{2}(\tilde{\Phi} \Phi),
$$


where $\tilde{\Phi} \equiv i \tau^{2} \Phi^{*}$, the Lagrangian (2.1) becomes

$$
\begin{aligned}
\mathcal{L}_{c l} & =-\frac{1}{2} \operatorname{Tr}\left(W_{\mu \nu} W^{\mu \nu}\right)-\frac{1}{4} B_{\mu \nu} B^{\mu \nu}+\frac{1}{4} \operatorname{Tr}\left(D_{\mu} M D^{\mu} M^{\dagger}\right) \\
& -\frac{\lambda}{4}\left(\frac{1}{2} \operatorname{Tr}\left(M M^{\dagger}\right)-\frac{\mu^{2}}{\lambda}\right)^{2} .
\end{aligned}
$$

The covariant derivative acting on the matrix $M$ is

$$
D_{\mu} M=\partial_{\mu} M+i g W_{\mu} M-i \frac{g^{\prime}}{2} M B_{\mu} \tau^{3}
$$

While the fields $\Phi$ and $\tilde{\Phi}$ transform exactly in the same way under the $S U(2)_{W}$ symmetry, they transform oppositely under the $U(1)_{Y}$ symmetry. This is reflected in the explicit presence of the $\tau^{3}$ matrix in the covariant derivative (2.7). Let us also recall that the ungauged Higgs Lagrangian has a global $S U(2)_{L} \times S U(2)_{R}$ symmetry, the so-called custodial symmetry. The custodial symmetry is explicitly broken once the gauge interactions are turned on.

The matrix $M$ can be written in terms of the physical Higgs field $H$ and the unphysical Goldstone bosons $\phi^{a}$ as

$$
M(x)=(v+H(x)) \Sigma(x), \quad \Sigma(x)=\exp \left(i \frac{\vec{\phi} \cdot \vec{\tau}}{v}\right)
$$

where $v=\sqrt{\mu^{2} / \lambda}$ is the vacuum expectation value. Using the non linear representation for the Higgs sector one then may write the Higgs Lagrangian as

$$
\mathcal{L}_{H i g g s}=\frac{1}{2} \partial_{\mu} H \partial^{\mu} H+\frac{(v+H)^{2}}{4} \operatorname{Tr}\left(D_{\mu} \Sigma D^{\mu} \Sigma^{\dagger}\right)-\frac{\lambda}{4}(v+H)^{4}+\frac{\mu^{2}}{2}(v+H)^{2} .
$$

The linear representation of the Higgs sector and the non-linear one are physically equivalent, and give the same physical answers. However, the last one is more suited to study the model in the strongly interacting limit $\lambda \rightarrow \infty$. In that limit the Higgs mass, $M_{H}=\sqrt{2 \lambda v^{2}}$, becomes large, and the Higgs field can be integrated out. Then the effective Lagrangian of the electroweak theory reduces at tree level to

$$
\mathcal{L}_{e f f}=-\frac{1}{2} \operatorname{Tr}\left(W_{\mu \nu} W^{\mu \nu}\right)-\frac{1}{4} B_{\mu \nu} B^{\mu \nu}+\frac{v^{2}}{4} \operatorname{Tr}\left(D_{\mu} \Sigma D^{\mu} \Sigma^{\dagger}\right) .
$$

That is, the low energy effective theory for the bosonic sector of the electroweak model is a gauged non-linear sigma model. This effective theory is non-renormalizable. The nonrenormalizability of the effective theory indicates the sensibility of the model to an ultraviolet cutoff, which here is interpreted as $M_{H}$. Beyond tree level, loop corrections associated with virtual Higgs-boson exchange lead to additional effective interactions. Those will not be considered here, anyway, since the study in this article will be restricted to thermal one-loop effects.

In the unitary gauge, that is, in the gauge where all the unphysical Goldstone bosons are eaten by the gauge fields (i.e. where $\Sigma=1$ ), one can read off the masses of the physical gauge fields from Eq. (2.10). The fields $W_{\mu}^{+}, Z_{\mu}$ and $A_{\mu}$ are defined as 


$$
\begin{aligned}
W_{\mu}^{\mp} & =\frac{1}{\sqrt{2}}\left(W_{\mu}^{1} \pm i W_{\mu}^{2}\right), \\
Z_{\mu} & =\cos \theta_{W} W_{\mu}^{3}-\sin \theta_{W} B_{\mu}, \\
A_{\mu} & =\sin \theta_{W} W_{\mu}^{3}+\cos \theta_{W} B_{\mu},
\end{aligned}
$$

where $\theta_{W}$ is the Weinberg angle, $\tan \theta_{W}=g^{\prime} / g$. The masses of those fields are

$$
M_{W}=\frac{v g}{2}, \quad M_{Z}=\frac{v}{2} \sqrt{g^{2}+g^{\prime 2}},
$$

while the mass of the photon is $M_{\gamma}=0$. At tree level the masses of the gauge fields obey the relation

$$
\rho \equiv\left(M_{W} / M_{Z} \cos \theta_{W}\right)^{2}=1
$$

as a consequence of the custodial symmetry.

Let us finally recall that the electric charge is defined as

$$
e=\frac{g g^{\prime}}{\sqrt{g^{2}+g^{\prime 2}}} .
$$

\section{THE BACKGROUND FIELD METHOD AND THE STUECKELBERG FORMALISM}

In this Section the background field method (BFM) [11] and the Stueckelberg formalism [12] for the gauged non-linear sigma model (2.10) are described. Those formalisms have already been used in the context of the electroweak model [13].

According to the BFM one has to split all fields into background and quantum pieces. The background fields are solutions of the classical equations of motion. In the present case one defines an additive splitting for the gauge fields, thus

$$
W_{\mu}(x)=\bar{W}_{\mu}(x)+w_{\mu}(x), \quad B_{\mu}(x)=\bar{B}_{\mu}(x)+b_{\mu}(x) .
$$

The background gauge fields have been represented by capital letters with a bar, while the quantum ones are denoted by lower case letters. The Goldstone fields are split multiplicatively, thus

$$
\Sigma(x)=\xi(x) h(x) \xi(x), \quad \bar{\Sigma}=\xi(x) \xi(x),
$$

where $\xi$ is a unitary matrix, $\xi \xi^{\dagger}=1$. The background field $\bar{\Sigma}$ and the quantum field $h$ are written in terms of background and quantum Goldstone fields, $\bar{\phi}$ and $\phi$, respectively, as $\bar{\Sigma}=\exp \left(i \bar{\phi}^{a} \tau^{a} / v\right)$ and $h=\exp \left(i \phi^{a} \tau^{a} / v\right)$.

After the splitting of fields is done, the Lagrangian (2.10) is separately invariant under background and quantum gauge transformations. Let us see what the behavior of the fields is under those two different kinds of gauge transformations.

Under background gauge transformations the background field $\bar{\Sigma}$ transform as follows 


$$
\bar{\Sigma}^{\prime}(x)=U_{W}(x) \Sigma(x) U_{Y}(x)
$$

where

$$
U_{W}(x)=\exp \left(i g \frac{\vec{\theta}_{W} \cdot \vec{\tau}}{2}\right), \quad U_{Y}(x)=\exp \left(i g^{\prime} \frac{\theta_{Y} \tau^{3}}{2}\right)
$$

The field $\xi$ behaves under the same transformation as

$$
\xi^{\prime}(x)=U_{W}(x) \xi(x) V^{\dagger}(x)=V(x) \xi(x) U_{Y}(x),
$$

and $V(x)$ is a unitary matrix which depends on $U_{W}, U_{L}$ and $\xi$. The background gauge fields transform as

$$
\begin{aligned}
\bar{W}_{\mu}^{\prime}(x) & =U_{W}(x) \bar{W}_{\mu}(x) U_{W}^{\dagger}(x)-\frac{i}{g} U_{W}(x) \partial_{\mu} U_{W}^{\dagger}(x), \\
\bar{B}_{\mu}^{\prime}(x) & =\bar{B}_{\mu}(x)+\partial_{\mu} \theta_{Y}(x)
\end{aligned}
$$

while under the same transformation, the quantum gauge fields behave as

$$
w_{\mu}^{\prime}(x)=U_{W}(x) w_{\mu}(x) U_{W}^{\dagger}(x), \quad b_{\mu}^{\prime}(x)=b_{\mu}(x), \quad h^{\prime}(x)=V(x) h(x) V^{\dagger}(x) .
$$

Under infinitesimal quantum gauge transformations the background fields remain invariant

$$
\delta \bar{W}_{\mu}(x)=0, \quad \delta \bar{B}_{\mu}(x)=0, \quad \delta \bar{\Sigma}(x)=0
$$

while the quantum gauge fields behave as

$$
\delta w_{\mu}^{a}=-\left(\partial_{\mu} \alpha_{w}^{a}-g \epsilon^{a b c}\left(\bar{W}_{\mu}^{b}+w_{\mu}^{b}\right) \alpha_{w}^{c}\right), \quad \delta b_{\mu}=\partial_{\mu} \alpha_{y} .
$$

The quantum Goldstone field transform as

$$
h^{\prime}(x)=V_{w}(x) h(x) V_{y}(x)
$$

where

$$
V_{w}(x)=\xi^{\dagger}(x) \exp \left(i g \frac{\vec{\alpha}_{w} \cdot \vec{\tau}}{2}\right) \xi(x), \quad V_{y}(x)=\xi(x) \exp \left(\frac{\alpha_{y} \tau^{3}}{2}\right) \xi^{\dagger}(x)
$$

In the spirit of the BFM the Lagrangian $\mathcal{L}_{\text {eff }}$ is expanded around the classical fields, keeping terms which are quadratic in the quantum fluctuations

$$
\mathcal{L}_{e f f}\left(W_{\mu}, B_{\mu}, \Sigma\right)=\mathcal{L}_{e f f}^{(0)}\left(\bar{W}_{\mu}, \bar{B}_{\mu}, \bar{\Sigma}\right)+\mathcal{L}_{e f f}^{(2)}\left(\bar{W}_{\mu}, \bar{B}_{\mu}, \bar{\Sigma}, w_{\mu}, b_{\mu}, \phi\right)+\ldots
$$

The linear terms in the quantum fluctuations vanish if one imposes the classical equations of motion for the background fields. To derive the one-loop effective action one has to integrate out the quantum fields appearing in $\mathcal{L}_{\text {eff }}^{(2)}$, adding the corresponding quantum gauge-fixing and quantum Faddeev-Popov terms. The functional integral can be done since the dependence on the fields is only quadratic. The background fields which appear in $\mathcal{L}_{\text {eff }}^{(2)}$ 
only act as sources for the generation of vertex functions in the effective action. If instead of making the functional integral one chooses a diagrammatic approach to get the one-loop effective action, then one only needs to consider Feynman diagrams with quantum fields running inside the loop, while the background fields only generate external vertices.

One of the advantages of using the BFM in a gauge field theory is that it gives a gauge invariant effective action in the background fields right away. To do so one only needs to fix the gauge of the quantum fields in a way invariant under the background gauge transformations. Then the associated Faddeev-Popov operators are also invariant under background gauge transformations.

In the BFM it is possible to fix the background and quantum gauges independently. In our case, and to simplify the computations, it is convenient to choose the unitary gauge for the background fields. Then the background Goldstone fields disappear completely from the Lagrangian, since they are eaten by the background gauge fields to become massive. In order to do so it is convenient to use the Stueckelberg formalism [12]. The Stueckelberg formalism allows one to eliminate the background Goldstone fields from the Lagrangian, thus it is equivalent to choosing the unitary background gauge. As will become clear, after performing a Stueckelberg transformation the fields $\bar{\Sigma}$ and $\xi$ are mapped to the unit matrix, and anything else remains unaffected. To see how this can be done, let us consider the following term of the Lagrangian (2.10)

$$
\frac{v^{2}}{4} \operatorname{Tr}\left(D_{\mu}(\xi h \xi) D^{\mu}(\xi h \xi)^{\dagger}\right) .
$$

The covariant derivative appearing in Eq. (3.14) can be written as

$$
D_{\mu}(\xi h \xi)=\xi\left(\partial_{\mu} h+\xi^{\dagger} \nabla_{\mu}^{W} \xi h-h \xi \nabla_{\mu}^{B} \xi^{\dagger}\right) \xi,
$$

where

$$
\begin{aligned}
& \xi^{\dagger} \nabla_{\mu}^{W} \xi=\xi^{\dagger}\left(\partial_{\mu} \xi+i g\left(\bar{W}_{\mu}+w_{\mu}\right) \xi\right), \\
& \xi \nabla_{\mu}^{B} \xi^{\dagger}=\xi\left(\partial_{\mu} \xi^{\dagger}+i g^{\prime}\left(\bar{B}_{\mu}+b_{\mu}\right) \frac{\tau^{3}}{2} \xi^{\dagger}\right) .
\end{aligned}
$$

Then if one performs the Stueckelberg transformation

$$
\begin{gathered}
\bar{W}_{\mu}^{\prime}=\xi^{\dagger} \bar{W}_{\mu} \xi-\frac{i}{g} \xi^{\dagger} \partial_{\mu} \xi, \bar{B}_{\mu}^{\prime} \frac{\tau^{3}}{2}=\xi\left(\bar{B}_{\mu} \frac{\tau^{3}}{2}\right) \xi^{\dagger}-\frac{i}{g^{\prime}} \xi \partial_{\mu} \xi^{\dagger}, \\
w_{\mu}^{\prime}=\xi^{\dagger} w_{\mu} \xi, \quad b_{\mu}^{\prime} \frac{\tau^{3}}{2}=\xi\left(b_{\mu} \frac{\tau^{3}}{2}\right) \xi^{\dagger},
\end{gathered}
$$

and one writes the Lagrangian in terms of the primed fields, all the background Goldstone fields $\xi$ disappear completely! It is easy to check that after the transformation (3.18-3.19) is done, Eq. (3.14) becomes

$$
\frac{v^{2}}{4} \operatorname{Tr}\left(D_{\mu}^{\prime} h D^{\prime \mu} h^{\dagger}\right)
$$


It has also to be pointed out that the Jacobian of the change of variables (3.19) is one at one-loop order.

The Stueckelberg transformation simplifies drastically the one-loop computations. Once the computation is finished, the Stueckelberg transformation has to be inverted to recover the presence of the background Goldstone bosons in the final one-loop effective action.

In order to simplify the notation from now on I will omit the primes in the fields, keeping in mind that the transformation has to be inverted at the end of the computation.

To integrate out the quantum fields a quantum gauge fixing condition invariant under the background gauge transformation has to be given. In the unitary background gauge the gauge fixing condition for the quantum fields is chosen as

$$
\mathcal{L}_{g f}^{(2)}=-\frac{1}{a_{w}} \operatorname{Tr}\left(\bar{D}_{W}^{\mu} w_{\mu}-\frac{1}{4} a_{w} g v \phi\right)^{2}-\frac{1}{2 a_{b}}\left(\partial^{\mu} b_{\mu}+\frac{1}{2} a_{b} g^{\prime} v \phi_{3}\right)^{2},
$$

where $a_{w}$ and $a_{b}$ are the gauge fixing parameters. These gauge fixing terms are chosen such as to cancel the unwanted pieces $\partial^{\mu} b_{\mu} \phi_{3}$ and $\operatorname{Tr}\left(\partial^{\mu} w_{\mu} \phi\right)$ in $\mathcal{L}_{\text {eff }}^{(2)}$. The form of the gauge fixing term in an arbitrary background gauge can be obtained by inverting the Stueckelberg transformation.

The Faddeev-Popov terms associated to the gauge fixing (3.21) are computed as usual. Finally, the complete one-loop quantum Lagrangian reads in Minkowski space

$$
\begin{aligned}
\mathcal{L}_{\text {eff }}^{(2)}+\mathcal{L}_{g f}^{(2)}+\mathcal{L}_{F P}^{(2)} & =\operatorname{Tr}\left(w_{\mu}\left(g^{\mu \nu} \bar{D}_{W}^{2}+\frac{1-a_{w}}{a_{w}} \bar{D}_{W}^{\mu} \bar{D}_{W}^{\nu}+2 i g \bar{W}^{\mu \nu}\right) w_{\nu}\right) \\
& +\frac{1}{2} b_{\mu}\left(g^{\mu \nu} \partial^{2}+\frac{1-a_{b}}{a_{b}} \partial^{\mu} \partial^{\nu}\right) b_{\nu} \\
& +M_{W}^{2} \operatorname{Tr}\left(w_{\mu} w^{\mu}\right)+\frac{M_{B}^{2}}{2} b_{\mu} b^{\mu}-g g^{\prime} v^{2} w_{\mu}^{3} b^{\mu} \\
& +\frac{1}{4} \operatorname{Tr}\left(\bar{d}_{\mu} \phi\right)^{2}-\frac{1}{4} \operatorname{Tr}\left[\bar{\Delta}_{\mu}, \phi\right]^{2}-\frac{a_{w} M_{W}^{2}}{4} \operatorname{Tr} \phi^{2}-\frac{a_{b} M_{B}^{2}}{2} \phi_{3}^{2} \\
& +2 v \operatorname{Tr}\left(\left(g w_{\mu}-g^{\prime} b_{\mu} \frac{\tau^{3}}{2}\right) \bar{\Gamma}^{\mu} \phi\right) \\
& -\eta_{a}^{\dagger}\left(\delta^{a b} \bar{D}_{W}^{2}+\delta^{a b} a_{w} M_{W}^{2}\right) \eta_{b}
\end{aligned}
$$

where $M_{B}^{2}=g^{\prime 2} v^{2} / 4$ and

$$
\begin{aligned}
\bar{D}_{W}^{\mu} & =\partial^{\mu}+i g\left[\bar{W}^{\mu},\right] \\
\bar{d}_{\mu} \phi & =\partial_{\mu} \phi+\left[\bar{\Gamma}_{\mu}, \phi\right] \\
\bar{\Gamma}_{\mu} & =\frac{i}{2}\left(g \bar{W}_{\mu}+g^{\prime} \bar{B}_{\mu} \frac{\tau^{3}}{2}\right) \\
\bar{\Delta}_{\mu} & =\frac{i}{2}\left(g \bar{W}_{\mu}-g^{\prime} \bar{B}_{\mu} \frac{\tau^{3}}{2}\right) .
\end{aligned}
$$

The ghost fields $\eta_{a}$ are associated to the $w_{\mu}^{a}$ quantum fields. Since the ghost associated to the $b_{\mu}$ field does not couple to any background external field, it has been omitted in Eq. (3.22). 
The one-loop Lagrangian (3.22) is written in the unitary background gauge. It can be obtained in an arbitrary background gauge by inverting the Stueckelberg transformation. However, it is much simpler to integrate out the quantum fields first, and invert the transformation afterwards to obtain the one-loop effective action in a general background gauge. That is what it will be done in the following Section.

\section{ONE-LOOP THERMAL EFFECTIVE ACTION FOR SOFT MODES}

\section{A. Unitary Background Gauge}

In this Section one-loop thermal effects are derived using the BFM Lagrangian (3.22) in an arbitrary quantum gauge $a_{w}, a_{b} \neq 0, \infty$. In those gauges the quantum fields $w_{\mu}^{a}, b_{\mu}$, $\phi^{a}$ and also the ghosts are massive. Only thermal effects will be studied, and the $T=0$ results will not be taken into account. I will actually take profit of results already derived in the literature to get the thermal effective action. Those results were obtained using the imaginary time formalism.

The analysis will be restricted to soft background fields $\bar{W}_{\mu}, \bar{Z}_{\mu}$ and $\bar{A}_{\mu}$. Those fields can only be soft if their respective masses are also soft, thus $M_{W}, M_{Z} \ll T$. This, in turn, implies that $g^{2}, g^{2} \ll\left(T^{2} / v^{2}\right)$.

For soft background fields the leading thermal corrections arise when the internal quantum fields are hard [4], that is, of energy $\sim T$. If one neglects corrections of order $M_{W} / T$ and $M_{Z} / T$ in the final answers, then it is possible to neglect those masses for the quantum fields. In other words, for hard quantum fields the terms $\partial^{2}$ of the Lagrangian are of the order $T^{2}$, which are dominant as compared to the terms $M_{W, B}^{2}$, which therefore will be neglected.

The computation simplifies once the masses of the quantum fields are neglected. One encounters here the same one-loop thermal amplitudes, the HTL's, which appear in the BFM of Yang-Mills theories [4], as well as in the non-linear sigma model in the presence of external background sources [10]. There are also new types of vertices in (3.22), which do not appear in the BFM studies of the previous mentioned theories: those which couple quantum gauge fields and quantum Goldstone bosons. However, a power counting analysis shows that the one-loop thermal corrections generated by those vertices are subleading as compared to the HTL's, and therefore they will be neglected.

The one-loop thermal effective action for soft background gauge fields is then a combination of the one which appears in a Yang-Mills theory and the one in the non-linear sigma model in the presence of external sources. I refer to the literature to see how those effective actions are computed [4], [6], [10]. By translating those results to our case one finds the following one-loop thermal effective action

$$
\begin{aligned}
S_{e f f}+\delta S_{e f f, T} & =\int d^{4} x\left\{-\frac{1}{2} \operatorname{Tr}\left(\bar{W}_{\mu \nu} \bar{W}^{\mu \nu}\right)-\frac{1}{4} \bar{B}_{\mu \nu} \bar{B}^{\mu \nu}+\frac{v^{2}(T)}{4} \operatorname{Tr}\left(g \bar{W}^{\mu}-g^{\prime} \bar{B}^{\mu} \frac{\tau^{3}}{2}\right)^{2}\right\} \\
& -\frac{T^{2}}{6} \int \frac{d \Omega_{\mathbf{q}}}{4 \pi} \int d^{4} x d^{4} y \operatorname{Tr}\left(\bar{\Gamma}_{\mu \lambda}(x)<x\left|\frac{Q^{\mu} Q_{\nu}}{-(Q \cdot \bar{d})^{2}}\right| y>\bar{\Gamma}^{\nu \lambda}(y)\right)
\end{aligned}
$$




$$
+\frac{g^{2} T^{2}}{3} \int \frac{d \Omega_{\mathbf{q}}}{4 \pi} \int d^{4} x d^{4} y \operatorname{Tr}\left(\bar{W}_{\mu \lambda}(x)<x\left|\frac{Q^{\mu} Q_{\nu}}{-\left(Q \cdot \bar{D}_{W}\right)^{2}}\right| y>\bar{W}^{\nu \lambda}(y)\right)
$$

where $\bar{W}_{\mu \nu}, \bar{B}_{\mu \nu}$ are the field strengths of the corresponding background gauge fields, and

$$
\begin{aligned}
v(T) & =v\left(1-\frac{1}{12} \frac{T^{2}}{v^{2}}\right) \\
\bar{\Gamma}_{\mu \nu} & =\partial_{\mu} \bar{\Gamma}_{\nu}-\partial_{\nu} \bar{\Gamma}_{\mu}+\left[\bar{\Gamma}_{\mu}, \bar{\Gamma}_{\nu}\right]
\end{aligned}
$$

and $Q=(i, \mathbf{q})$ is a null vector $Q^{2}=0$. The angular integral in (4.1) is done over all directions of the three dimensional unit vector $\mathbf{q}$.

Let me remind the meaning of each term of Eq. (4.1). The two first terms are the kinetic pieces for the soft background gauge fields. The last piece in Eq. (4.1) is the HTL effective action for the non-Abelian gauge field $\bar{W}_{\mu}$, and it is generated by considering the one-loop thermal effects of the hard quantum gauge field $w_{\mu}^{a}$, and the quantum ghosts $\eta^{a}$ 画. The third and fourth terms in Eq. (4.1) arise after considering the one-loop thermal effects of the hard quantum Goldstone bosons $\phi^{a}$, (see Section III of Ref. [10 with the following identifications: $F_{\mu}^{R}=-g \bar{W}_{\mu}, F_{\mu}^{L}=-g^{\prime} \bar{B}_{\mu} \frac{\tau^{3}}{2}$, and $\xi=\xi^{\dagger}=1$.).

\section{B. Inverting the Stueckelberg transformation}

The thermal effective action (4.1) is given in the unitary background gauge. To recover the presence of the background Goldstone bosons in the effective action the Stueckelberg transformation (3.18) has to be inverted. Recall that the gauge fields which appear in Eq. (4.1) are the primed fields of Eq. (3.18).

After the inversion of the Stueckelberg transformation one gets the effective action

$$
\begin{aligned}
S_{e f f}+\delta S_{e f f, T} & =\int d^{4} x\left\{-\frac{1}{2} \operatorname{Tr}\left(\bar{W}_{\mu \nu} \bar{W}^{\mu \nu}\right)-\frac{1}{4} \bar{B}_{\mu \nu} \bar{B}^{\mu \nu}+\frac{v^{2}(T)}{4} \operatorname{Tr}\left(\bar{D}_{\mu} \bar{\Sigma} \bar{D}^{\mu} \bar{\Sigma}^{\dagger}\right)\right\} \\
& -\frac{T^{2}}{6} \int \frac{d \Omega_{\mathbf{q}}}{4 \pi} \int d^{4} x d^{4} y \operatorname{Tr}\left(\bar{\Gamma}_{\mu \lambda}(x)<x\left|\frac{Q^{\mu} Q_{\nu}}{-(Q \cdot \bar{d})^{2}}\right| y>\bar{\Gamma}^{\nu \lambda}(y)\right) \\
& +\frac{g^{2} T^{2}}{3} \int \frac{d \Omega_{\mathbf{q}}}{4 \pi} \int d^{4} x d^{4} y \operatorname{Tr}\left(\bar{W}_{\mu \lambda}(x)<x\left|\frac{Q^{\mu} Q_{\nu}}{-\left(Q \cdot \bar{D}_{W}\right)^{2}}\right| y>\bar{W}^{\nu \lambda}(y)\right) .
\end{aligned}
$$

where now the $\bar{W}^{\mu}$ field which enters in $\bar{W}^{\mu \nu}$ and $\bar{D}_{W}$ is the right hand side of Eq. (3.18), and the $\bar{\Gamma}_{\mu}$ field entering in $\bar{\Gamma}_{\mu \nu}$ and in $\bar{d}$ is

$$
\bar{\Gamma}_{\mu}=\frac{1}{2}\left(\xi^{\dagger} \bar{\nabla}_{\mu}^{W} \xi+\xi \bar{\nabla}_{\mu}^{B} \xi^{\dagger}\right)=\frac{1}{2}\left(\xi^{\dagger}\left(\partial_{\mu} \xi+i g \bar{W}_{\mu} \xi\right)+\xi\left(\partial_{\mu} \xi^{\dagger}+i g^{\prime} \bar{B}_{\mu} \frac{\tau^{3}}{2} \xi^{\dagger}\right)\right) .
$$

Equation (4.4) gives the one-loop effective action for soft background modes written in a general background gauge as a function of the background gauge fields and the background would-be Goldstone bosons. 


\section{THERMAL MASSES FOR SOFT GAUGE FIELDS}

The one-loop thermal effective action derived in Sec. IV describes gauge invariant mass terms for the gauge bosons $W_{\mu}^{+}, Z_{\mu}$ and $A_{\mu}$. In order to read off the thermal corrections to those masses from the effective action it is convenient to use the unitary gauge, where the unphysical Goldstone bosons are not present.

Let us consider the effective action (4.1) in the static limit. In the static limit the nonlocal terms of Eq.(4.1) become local. Apart from the kinetic terms for the gauge fields the Lagrangian reads

$$
\delta \mathcal{L}_{\text {eff } f, T}^{\text {static }}=\frac{v^{2}(T)}{4} \operatorname{Tr}\left(g \bar{W}^{\mu}-g^{\prime} \bar{B}^{\mu} \frac{\tau^{3}}{2}\right)^{2}+\frac{T^{2}}{12} \operatorname{Tr}\left(g \bar{W}_{0}+g^{\prime} \bar{B}_{0} \frac{\tau^{3}}{2}\right)^{2}+\frac{2 g^{2} T^{2}}{3} \operatorname{Tr}\left(\bar{W}_{0}\right)^{2} .
$$

Let us remark that the thermal corrections to the two first terms of (5.1) were obtained by integrating out the hard would-be quantum Goldstone bosons.

If one expresses Eq. (5.1) in terms of the physical fields $\bar{W}^{+}, \bar{Z}_{\mu}$ and $\bar{A}_{\mu}$, one obtains

$$
\begin{aligned}
\delta \mathcal{L}_{\text {eff }, T}^{\text {static }} & =\frac{g^{2} v^{2}(T)}{4} \bar{W}_{\mu}^{+} \bar{W}^{-\mu}+\frac{\left(g^{2}+g^{\prime 2}\right) v^{2}(T)}{4} \bar{Z}_{\mu} \bar{Z}^{\mu} \\
& +\frac{g^{2} T^{2}}{12} \bar{W}_{0}^{+} \bar{W}^{-0}+\frac{\left(g^{2}+g^{2}\right) T^{2}}{24}\left(\left(\cos ^{2} \theta_{W}-\sin ^{2} \theta_{W}\right)^{2} \bar{Z}^{0} \bar{Z}_{0}\right. \\
& \left.+4 \cos ^{2} \theta_{W} \sin ^{2} \theta_{W} \bar{A}_{0} \bar{A}^{0}+4 \cos \theta_{W} \sin \theta_{W}\left(\cos ^{2} \theta_{W}-\sin ^{2} \theta_{W}\right) \bar{Z}^{0} \bar{A}_{0}\right) \\
& +\frac{2 g^{2} T^{2}}{3} \bar{W}_{0}^{+} \bar{W}^{-0}+\frac{g^{2} T^{2}}{3}\left(\cos ^{2} \theta_{W} \bar{Z}^{0} \bar{Z}_{0}+\sin ^{2} \theta_{W} \bar{A}_{0} \bar{A}^{0}+2 \cos \theta_{W} \sin \theta_{W} \bar{Z}^{0} \bar{A}_{0}\right)
\end{aligned}
$$

The longitudinal and transverse gauge modes get different thermal corrections to their masses. The thermal masses for the transverse modes are

$$
M_{W, t}^{2}(T)=\frac{g^{2} v^{2}(T)}{4}, \quad M_{Z, t}^{2}(T)=\frac{\left(g^{2}+g^{\prime 2}\right) v^{2}(T)}{4}, \quad M_{\gamma, t}^{2}(T)=0
$$

while for the longitudinal ones are

$$
\begin{aligned}
& M_{W, l}^{2}(T)=\frac{g^{2} v^{2}(T)}{4}+\frac{3 g^{2} T^{2}}{4}, \\
& M_{Z, l}^{2}(T)=\frac{\left(g^{2}+g^{\prime 2}\right) v^{2}(T)}{4}+\frac{\left(g^{2}+g^{\prime 2}\right) T^{2}}{12}\left(\cos ^{2} \theta_{W}-\sin ^{2} \theta_{W}\right)^{2}+\frac{2 g^{2} T^{2}}{3} \cos ^{2} \theta_{W} \\
& M_{\gamma, l}^{2}(T)=e^{2} T^{2} .
\end{aligned}
$$

To express the electric thermal mass of the photon in terms of the electric charge $e$, use of the relation (2.16) has been made. The above results agree with those computed in Ref. [8].

Let me point out that in this article it has been considered that $M_{W}, M_{Z} \ll T$, which implies $g^{2}, g^{\prime 2} \ll T^{2} / v^{2}$. It follows that the thermal corrections coming from integrating out the hard quantum Goldstone bosons are dominant as compared to those arising by integrating out the hard quantum gauge degrees of freedom. With this in mind, one can generally claim that while the transverse modes masses decrease with $T$, the longitudinal ones may increase with $T$, as a consequence of the Debye screening phenomena. It is also interesting to point out that while the transverse masses obey the relation (2.15), the longitudinal ones do not, once again, as a result of the thermal Debye screening. 


\section{CONCLUSIONS}

The cornerstone of the electroweak model is the spontaneous breaking of the gauge symmetry. The would-be Goldstone bosons associated to that breaking are eaten by the gauge fields to become massive, through the standard Higgs mechanism. If the Higgs mass is very large, one can remove the Higgs field from the action to finally obtain a low energy effective theory.

One of the advantages of the low energy theory is that at leading order is universal once the pattern of the $S U(2)_{W} \times U(1)_{Y}$ symmetry breaking is known. The model dependence only arises at one-loop level, through the dependence on the ultraviolet cutoff, and the matching conditions between the low and high energy theories.

In this article the one-loop computations have been done putting the natural ultraviolet cutoff $M_{H} \rightarrow \infty$. A finite value of $M_{H}$, with $T \ll M_{H}$, will introduce an explicit dependence of the thermal gauge boson masses on $M_{H}$. These corrections to Eqs. (5.3 5.6) would go as $\sim-T^{2} \exp \left(-M_{H} / T\right)$ and are therefore subleading.

Some general statements can be made:

- The would-be Goldstone bosons give a negative thermal contribution in the transverse or magnetic masses. This has been checked at the one-loop level.

- There is a Debye screening phenomena taking place, since the model contains charged particles. The charged particles yield a thermal contribution which increases the value of the longitudinal or electrical masses with $T$.

One should also note that if there were magnetic charges in the model, either elementary or composed objects, a dual Debye screening phenomena would take place [14], [15], and as a result the gauge transverse masses should increase with $T$. Those effects would not be computable in perturbation theory, though.

An important issue to be discussed is the one of resummation. In QCD resummation of HTL's is mandatory for soft gauge fields, as shown by using a power counting analysis [4]. In the broken phase of the electroweak model the same kind of arguments could be applied for the soft gauge fields: tree graphs and the one-loop thermal amplitudes described by the effective action (4.1) are equally important, and resummation should be done. The implementation of the resummation program in the electroweak model seems to be much more complicated than in QCD due to the presence of the would-be Goldstone bosons. However, an advantage of the electroweak model is that the perturbative approach should be free of infrared problems, contrary to what happens in QCD, due to the existence of thermal magnetic masses. I hope to report in a future project on the implementation of the resummation program in the electroweak theory.

\section{Acknowledgments:}

I am specially thankful to N. Rius for many helpful e-mail discussions in the course of this project. I have also benefited from useful comments of D. Espriu and D. Litim. This work has been supported by funds provided by the CICYT contract AEN95-0590, the CIRIT contract GRQ93-1047, and the NATO grant CRG-970055. 


\section{REFERENCES}

[1] T. Appelquist and C. Bernard, Phys. Rev. D22, 200 (1980); A. Longhitano, ibid, D22, 1166 (1980); Nucl. Phys. B188, 118 (1981).

[2] L. Dolan and R. Jackiw, Phys. Rev. D9, 3320 (1974); S. Weinberg, ibid D9, 3357 (1974).

[3] M. Le Bellac, "Thermal Field Theory", Cambridge University Press, Cambridge 1996.

[4] R. D. Pisarski, Phys. Rev. Lett. 63, 1129 (1989); E. Braaten and R. Pisarski, Nucl. Phys. B337, 569 (1990); J. Frenkel and J. C. Taylor, Nucl. Phys. B334, 199 (1990).

[5] R. Efraty and V. P. Nair, Phys. Rev. D47, 5601 (1993); R. Jackiw and V. P. Nair, ibid D48, 4991 (1993); J. P. Blaizot and E. Iancu, Nucl. Phys. B390, 589 (1993); R. Jackiw, Q. Liu, and C. Lucchesi, Phys. Rev. D49, 6787 (1994); P. F. Kelly, Q. Liu, C. Lucchesi, and C. Manuel, ibid D50, 4209 (1994).

[6] J. C. Taylor and S. M. H. Wong, Nucl. Phys. B346 115 (1990); E. Braaten and R. Pisarski, Phys. Rev. D45 (1992) R1827.

[7] P. Binetruy and M. K. Gaillard, Phys. Rev. D32, 931 (1985); J. Gasser and H. Leutwyler, Phys. Lett. B184, 83 (1987); P. Gerber and H. Leutwyler, Nucl. Phys. B321, 387 (1989); M. Dey, V. L. Eletsky, and B. L. Ioffe, Phys. Lett. B252, 620 (1990); A. Bochkarev and J. Kapusta, Phys. Rev. D54, 4006 (1990); R. D. Pisarski and M. Tytgat, ibid D54, 2989 (1996); D. Toublan, ibid D56, 5629 (1997).

[8] M. B. Gavela, O. Pène, N. Rius, and S. Vargas-Castrillón, LPTHE Orsay 96/36, FTUAM FEV 96/22, FTUV 97-73, IFIC 97-105, hep-ph/9801244.

[9] R. D. Pisarski and M. Tytgat, Phys. Rev. Lett. 78, 3622 (1997).

[10] C. Manuel, hep-ph/9710208 to be published in Phys. Rev. D

[11] B. S. DeWitt, Phys. Rev. 162, 1195 (1967); L. F. Abbot, Nucl. Phys. B185, 33 (1985).

[12] E. C. Stueckelberg, Helv. Phys. Acta 11, 299 (1938); ibid, 30, 209 (1956); F. Jegerlehner and J. Fleisher, Acta Phys. Polon. B17, 709 (1986); C. Grosse-Knetter and R. Kogerler, Phys. Rev. D48, 2865 (1993).

[13] S. Dittmaier and C. Grosse-Knetter, Nucl. Phys. B459, 497 (1996).

[14] D. J. Gross, R. D. Pisarski, and L. G. Yaffe, Rev. Mod. Phys. 53, 43 (1981).

[15] C. Manuel, hep-ph/9612494 to be published in Ann. Phys. (N.Y.). 